\title{
Spontaneous emission enhancement in a microtube cavity with highly confined optical modes
}

\author{
Y.P. Rakovich *a, S. Balakrishnan ${ }^{\text {b }}$, Y. Gun'ko ${ }^{\text {b }}$, T.S. Perova ${ }^{\text {c }}$, A. Moore ${ }^{\text {c }}$, J.F. Donegan ${ }^{\text {a,d }}$ \\ ${ }^{a}$ Semiconductor Photonics Group, School of Physics, Trinity College Dublin, Dublin 2, Ireland; \\ ${ }^{\mathrm{b}}$ School of Chemistry, Trinity College Dublin, Dublin 2, Ireland; \\ ${ }^{c}$ Department of Electronic and Electrical Engineering, Trinity College Dublin, Dublin 2, Ireland; \\ ${ }^{\mathrm{d}}$ Principal Investigator CRANN Research Centre, TrinityCollege Dublin, Dublin 2, Ireland
}

\begin{abstract}
A detailed study of the photonic modes in microtube cavity of $\sim 7-8 \mu \mathrm{m}$ outer diameter is presented. We demonstrate a new route to the fabrication of individual microtubes with the maximum length of $200 \mu \mathrm{m}$, using a vacuum assisted wetting and filtration through a microchannel glass matrix. The microtubes were studied using micro-photoluminescence spectroscopy and luminescence lifetime imaging confocal microscopy. In the emission spectra of the microresonators we find periodic very narrow peaks corresponding to the whispering gallery modes of two orthogonal polarizations with quality factors upto 3200 at room temperature. In order to identify the peaks in the observed mode structure, we have adopted the boundary-value solution to the problem of scattering of electromagnetic waves by a dielectric microcylinder. A strong enhancement in photoluminescence decay rates at high excitation power suggest the occurrence of amplified spontaneous emission from a single microtube. The evanescent field in these photonic structures extends a couple of micrometers into the surroundings providing the possibility for efficient coupling to an external photonic device.
\end{abstract}

Keywords: microcavities, microtubes, whispering gallery modes, amplified spontaneous emission, FLIM imaging

\section{INTRODUCTION}

Optical microcavities, which confine the propagation of light in all three dimensions, are extensively studied due to their microscopic size (1-500 microns), high quality factor Q $\left(10^{2}-10^{9}\right)$ and the possibility of achieving low threshold lasing (less than $1 \mu \mathrm{W})^{1-8}$. These systems exhibit novel features arising from our ability to tailor the electro-magnetic field and the photon density of states in a prescribed manner, such as to concentrate and guide light on the micrometer scale, couple light between different components, or inhibit/enhance spontaneous emission. Such systems can be divided into two size regimes: for microcavities of size greater than 100 microns, the quality factors exceed $10^{6}$, the modes are tightly confined within the cavity and are spectrally close together. For structures of less than 100 microns and particularly for those less than 10 microns, the quality factors are smaller $\left(10^{4}\right.$ or less) and the modes are spectrally well spaced. However, it is the evanescent field of the cavity modes that extend many microns outside the cavity that make such structures interesting, in particular opening up the possibility of coupling microcavities to produce new photonic structures.

Among the microcavity systems which have been developed during the past 10-15 years, optical emitters with cylindrical symmetry which support whispering gallery modes (WGMs) have attracted particular interest. ${ }^{2-4}$ The resonantly enhanced optical response make these high-Q microcavities attractive as novel building blocks for photonic devices. The cylindrical cavity format is also compatible with a large variety of sensing modalities such as immunoassay and molecular diagnostic assay., ${ }^{9,10}$

Experimentally, the most widely studied configuration of thin-wall microtube cavities is the microcapillary filled with a highly-luminescent dye solution. ${ }^{3,4}$ The diameter (typically 50-200 $\mu \mathrm{m}$ ) and wall thickness of these microcapillaries can be controlled by the etching commercially-available glass samples in an HF-water solution. In the small-size regime (diameter $<10 \mu \mathrm{m}$ ), semiconductor microdisks or micropillars of finite height have been widely used as tools to control spontaneous emission and confine photons in three dimensions. ${ }^{2}$

*Yury.Rakovich@tcd.ie; phone (+)353 1896 1821; fax (+) 353 11-671-1759

ICONO 2007: Novel Photonics Materials; Optics and Optical Diagnostics of Nanostructures

edited by Oleg A. Aktsipetrov, Vladimir M. Shalaev, Sergey V. Gaponenko, Nikolay I. Zheludev

Proc. of SPIE Vol. 6728, 672806, (2007) · 0277-786X/07/\$18 - doi: 10.1117/12.752145

Proc. of SPIE Vol. 6728 672806-1 
The synthesis of tubular materials such as silica, magnesium oxide, titanium dioxide and zinc oxide has been reported. ${ }^{11}$ Along with these techniques the fabrication of micro-wire arrays in situ using electrochemical ${ }^{12-14}$ and electroless ${ }^{15-17}$ deposition in a porous template has proven to be a superior option for preparing more complex, higher aspect ratio 3-D microtube and microwire arrays. ${ }^{18}$ The formation of luminescent structures by incorporating rare-earth ions into a porous matrix using various techniques like ion-implantation,[19] electrochemical migration, ${ }^{20}$ or the spin-on technique ${ }^{21}$ has been demonstrated. However, the fabrication of small $(<10 \mu \mathrm{m}$ diameter $)$ high-Q cylindrical microcavities generally involves complex and time-consuming processing., ${ }^{2,22}$ Here we describe a new and very straightforward method for fabricating highly-luminescent small aluminosilicate microtubes of $\sim 6-7 \mu \mathrm{m}$ outer diameter, which are shown to act as optical cylindrical micro resonators.

\section{EXPERIMENTAL}

Tetraethylorthosilicate $\left(\mathrm{Si}\left(\mathrm{OC}_{2} \mathrm{H} 5\right) 4\right.$ or TEOS $)$ and $\mathrm{Al}(\mathrm{OC} 4 \mathrm{H} 9 \mathrm{sec}) 3$ have been purchased from Aldrich. The microchannel glass samples of $8-9 \mu \mathrm{m}$ and a thickness of about $0.05 \mathrm{~cm}$ have been provided by State Optical Institute, St. Petersburg.

In our fabrication approach, we took advantage of the well-developed sol-gel technique combined with the versatility of ordered porous membranes as templates. $\mathrm{Si}(\mathrm{OC} 2 \mathrm{H} 5) 4$ was first hydrolysed for $1 \mathrm{~h}$ at room temperature with a solution of $\mathrm{H} 2 \mathrm{O}, \mathrm{C} 2 \mathrm{H} 5 \mathrm{OH}$ and $\mathrm{HCl}$ in the molar ratios 1: 1: 0.0027 per mole of $\mathrm{Si}(\mathrm{OC} 2 \mathrm{H} 5) 4$ respectively. Then $\mathrm{Al}(\mathrm{OC} 4 \mathrm{H} 9 \mathrm{sec}) 3$ was added to this solution and the mixture was stirred for $15 \mathrm{~min}$ at $70^{\circ} \mathrm{C}$. The resultant homogenous mixture was hydrolysed by adding the mixed solution of $\mathrm{H} 2 \mathrm{O}, \mathrm{C} 2 \mathrm{H} 5 \mathrm{OH}$ and $\mathrm{HCl}$ in the molar ratio 4: 1: 0.011 per mole of alkoxide, respectively. Finally, 30 minutes stirring provided the conversion of this sol to $5 \mathrm{Al} 2 \mathrm{O} 3.95 \mathrm{SiO} 2(\mathrm{~mol} \%)$ alumosilicate gel. The sol, just before its gelation point was then placed on the top of the micro-channel glass samples and $30 \mathrm{mbar}-$ vacuumassisted filtration resulted in the formation of microtubes inside the channels. Following drying at room temperature for 1 day and further annealing at $500^{\circ} \mathrm{C}$ for 2 hours, the fabricated microtubes were isolated by mechanical destruction of the template. A sequential flow diagram of the procedure is depicted in Figure 1.

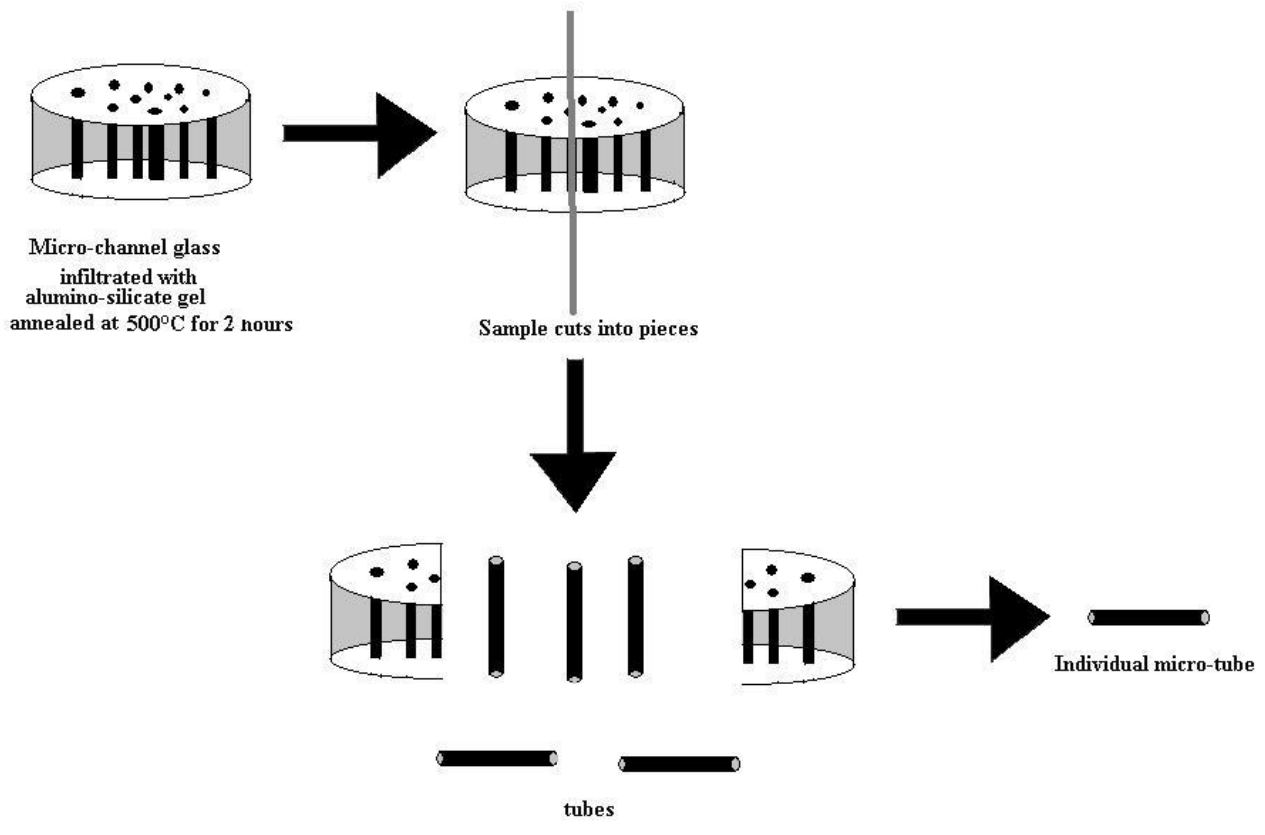

Fig. 1. A sequential flow diagram showing the drawing of individual microtubes from the microchannel glass matrix.

The steady-state photoluminescence (PL) spectra from both a single microtube and microtubes within the matrix were recorded in a backscattering geometry using a RENISHAW micro-Raman system $\left(1800 \mathrm{~mm}^{-1}\right.$ grating, $>1 \mathrm{~cm}^{-1}$ resolution) equipped with a microscope objective $(\times 100)$, a notch and plasma filters and a CCD camera. An $\operatorname{Ar}+$ laser (wavelength $514.5 \mathrm{~nm}$ ) was used as the excitation source. PL decays were measured using time-correlated single-photon counting (Time-Harp, PicoQuant). The samples were excited by $480 \mathrm{~nm}$ picosecond pulses generated by a PicoQuant, LDH-480 laser head controlled by a PDL-800B driver. The setup was operated at a $20-\mathrm{MHz}$ repetition rate with an 
overall time resolution of $\sim 150$ psec. Fluorescence lifetime images from the microtube were collected with the FLIM (fluorescence lifetime imaging microscopy) system (Microtime200 time-resolved confocal microscope system, PicoQuant) equipped with an Olympus IX71 inverted microscope. Lifetime maps were calculated on a pixel-by-pixel basis by fitting the lifetime of each pixel to the logarithm of the intensity. The FLIM system response was negligible compared with typical lifetimes of the aluminosilicate microtubes ( 3-10 ns). For the amplified spontaneous emission (ASE) experiments, PL decays at varying excitation powers were measured in the region of the selected photonic mode using a narrow-band filter. The excitation power was measured just before entering the objective using a calibrated power diode. The pump laser with its polarization parallel to the microtube axis was tightly focused (beam size $\sim 1 \mu \mathrm{m}$ ). The scanning electron microscopy (SEM), images of the samples were obtained using an FESEM (Hitachi S-4300 scanning electron microscope), which was operated at $5.0 \mathrm{kV}$ or $20 \mathrm{kV}$. The beam-specimen interaction produces a variety of signals including secondary electrons (SE), back-scattered electrons (BE) and X-rays. The low energy secondary electrons are collected to form the standard image and the high energy back-scattered electrons provide an image with good atomic number contrast. Samples for analysis were mounted on aluminium stubs and coated with gold.

\section{RESULTS AND DISCUSSION}

Cross-sectional Scanning Electron Microscopy (SEM) images of the microtubes are presented in Figure 2. From these images it can be seen that the microtubes are hollow. The images also show the uniform distribution of the microtubes inside the porous matrix. From the SEM pictures, we find that the microtubes have an outer diameter of 6-7 $\mu \mathrm{m}$ and an inner diameter of 2.7-3 $\mu \mathrm{m}$. The maximum length of a single microtube is $200 \mu \mathrm{m}$. Thus, the SEM images show the formation of high aspect ratio microtubes having smooth walled structures, ideal for photonic studies.
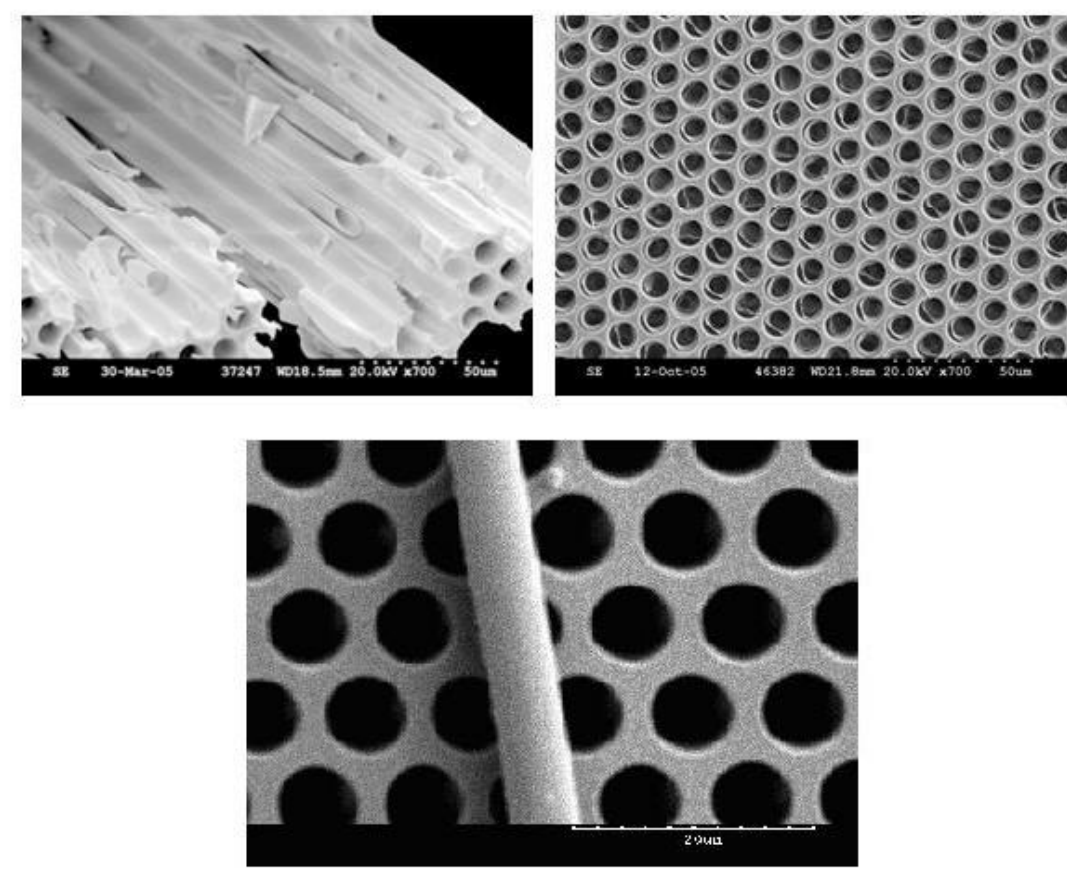

Fig. 2. Cross-sectional and plan view SEM images of the microtubes inside and outside the matrix.

The formation of alumosilicate microtubes by vacuum filtration of the gel can be explained by the fact that the cohesive driving forces for complete microchannel filling are substantially weaker than the adhesive forces to the pore walls. Similar phenomena have been reported for the formation of polymer nanotubes by wetting of ordered porous templates using a polymer melt. ${ }^{23}$

The thermal treatment of the samples resulted in highly emissive air-stable structures which display broad-band visible photoluminescence originating from carbon defects substituting for silicon in the microtube material. ${ }^{24}$ An elemental analysis of the annealed aluminosilicate gel showed carbon and hydrogen content of $0.32 \%$ and of $0.56 \%$ respectively. The PL spectrum of a single aluminosilicate microtube accommodated in the microporous glass substrate is presented in 
Figure 3a. The emission spectrum shows a broad luminescence band ranging from 520 to $800 \mathrm{~nm}$. The PL of a single aluminosilicate microtube shows a clear multi-exponential decay. The observed multi-exponential decay kinetics are consistent with a PL model that includes multiple emission pathways and is indicative of a broad lifetime distribution caused by the corresponding distribution of defect or trap states due to the amorphous nature of the microtube material (Figure 3b).
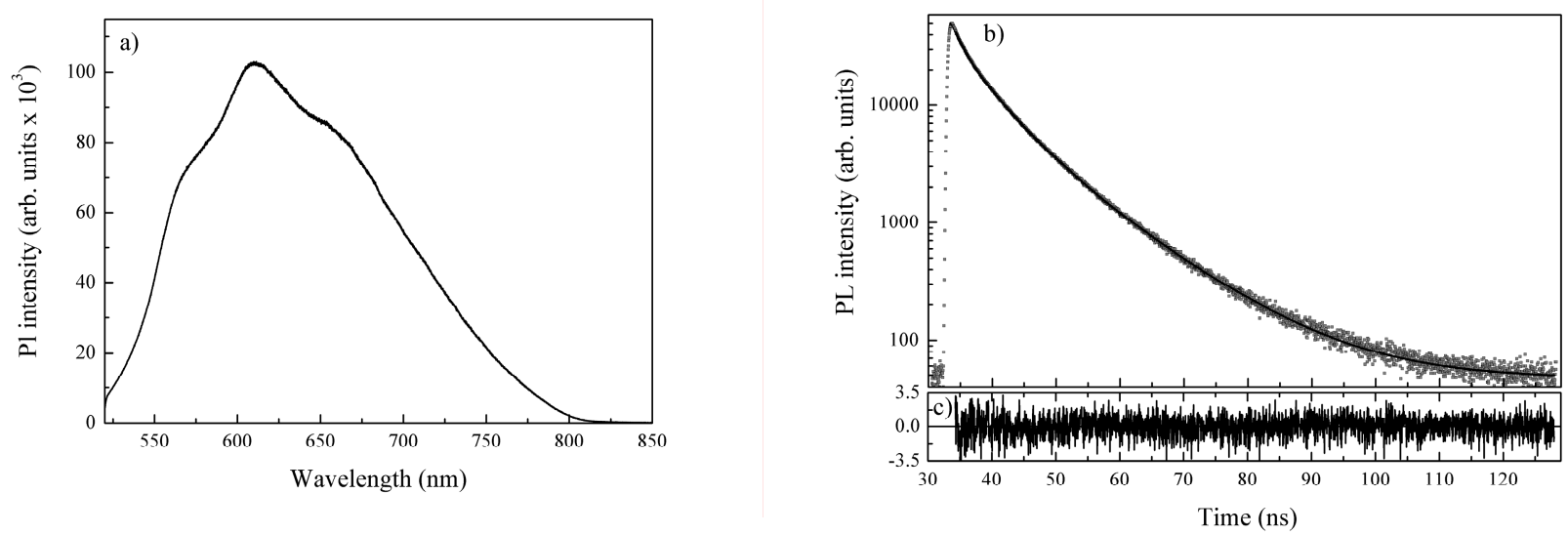

Fig. 3. (a) PL spectra and (b) time-dependent PL intensity decay of a single aluminosilicate microtube accommodated in a glass microchannel matrix with corresponding residuals (c).

In the case when fluorophores are embedded in nonuniform environments, it has been shown that PL decays can be best understood by a model of continuous distributions of decay times. ${ }^{25}$ In this case fitting procedures cannot distinguish sufficiently between, for example, a single Gaussian distribution of lifetimes and the sum of two exponentials or a bimodal Gaussian distribution and the sum of at least three exponentials. ${ }^{26,27}$ Thus, a description based on discrete lifetime components should only be regarded as truly representing discrete molecular states if supported by supplementary data. ${ }^{28}$ Therefore, to gain a better insight into the spatial distribution of lifetimes, the PL dynamics were evaluated from the FLIM images i.e. maps of two-dimensional in-plane variations of the PL decay times.

Each pixel in the FLIM image gives the lifetime at a particular position in space $(x, y)$ while monitoring the entire PL spectrum. The lifetime image of the array of aluminosilicate microtubes in a micro-porous glass matrix (Figure $4 a$ and $b$ ) clearly demonstrates the distribution of emitting species over the microtube cross-section, showing that the dominant emission is at the rim of the microtube. In both images, the histograms show lifetime distributions that consist of two maxima centered at $0.7 \mathrm{~ns}$ and $3.5 \mathrm{~ns}$ with the values of full width at half maximum FWHM $=0.5 \mathrm{~ns}$ and $2 \mathrm{~ns}$ respectively. Comparing the lifetime histogram obtained from the array (Figure 4c) with that measured from a single microtube, accommodated in the porous matrix, (Figure 4d) it is very clear that there is no deviation in lifetime within the ensemble of microtubes formed in the matrix. This demonstrates the high quality of the samples and the reproducibility of the optical parameters.

When separated from the matrix, a drastic modification in lifetime distribution has been observed in the FLIM image of the individual microtube cavity (Figure 5). Once again two maxima in the lifetime histogram of the "free standing" single microtube are present. However, in contrast to the embedded microtube, the longlived component centered at 10 ns is now dominant and is accompanied by a second smaller peak at 4 ns. The faster decay observed in the case of the embedded microtube implies that lifetimes of the emitting states are strongly reduced, thereby demonstrating the presence of highly-efficient nonradiative energy transfer from the microtube into the surrounding matrix. In contrast, when the microtube is separated from the matrix, the nonradiative pathways introduced by the martix are no longer competing with the radiative pathways, resulting in strong enhancement of lifetimes. The two-peak structure shown in Figures 4 and 5 implies that at least two different mechanisms are involved in the decay processes. The peak around $3 \mathrm{~ns}$ is almost unaltered in going from the matrix to the free microtube. It is therefore part of the bulk of the microtube material and is unaffected by the microcavity. The defect is likely to have a deep state with a highly localized wavefunction. 
On the other hand, there is a significant alteration to the short lifetime component. Its average value increases by an order of magnitude. As we will see later, the microtube microcavity structure has a large evanescent field. While in the matrix there is very strong coupling of this field to the matrix and this is removed once the microtube is free from the matrix. Very efficient transfer in a Förster energy transfer mechanism will result in nonradiative decay processes. ${ }^{29}$ This type of energy transfer can occur through dipole-dipole interactions between an emitter in the glass and the matrix surface and is strongly dependent on distance between them. The large evanescent field of the microtube will enhance the transfer rate thereby producing the short component in the lifetime of the PL within the matrix.

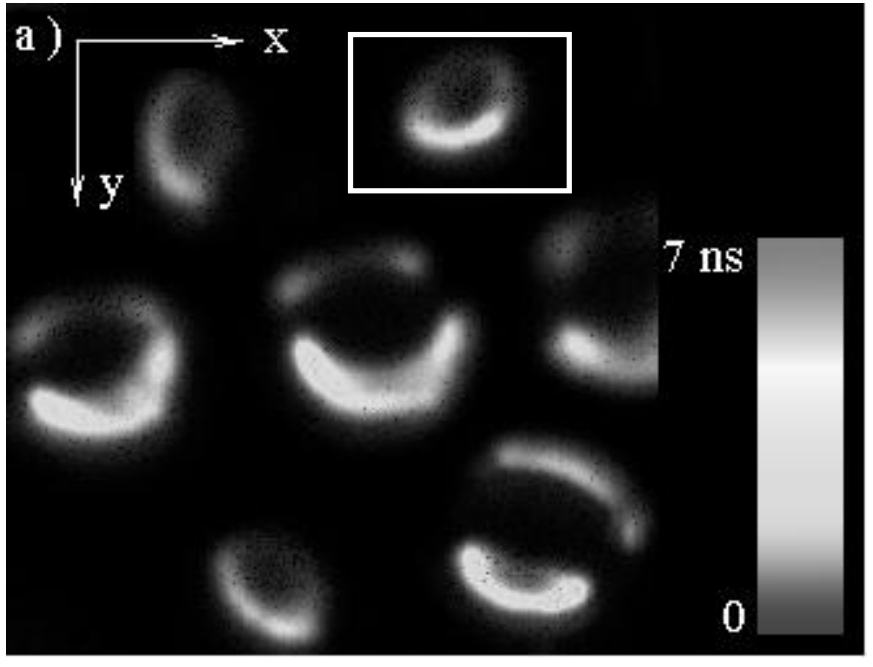

c)

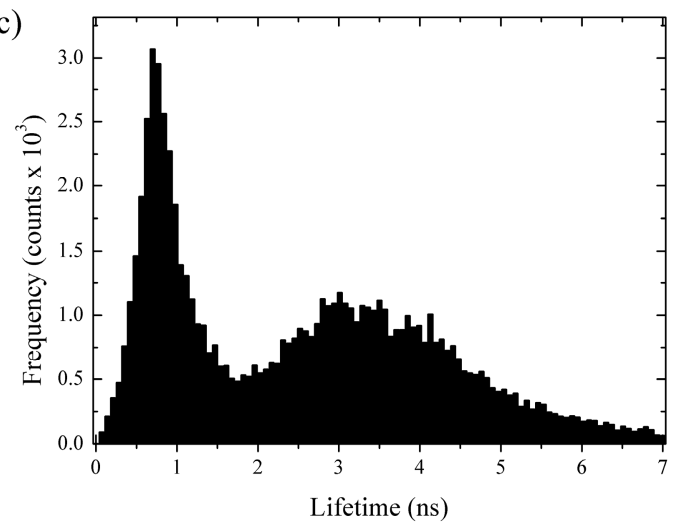

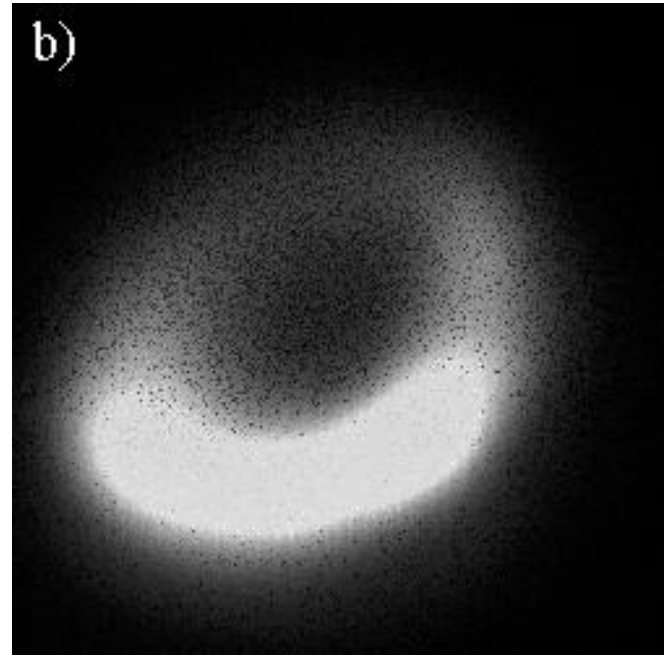

d)

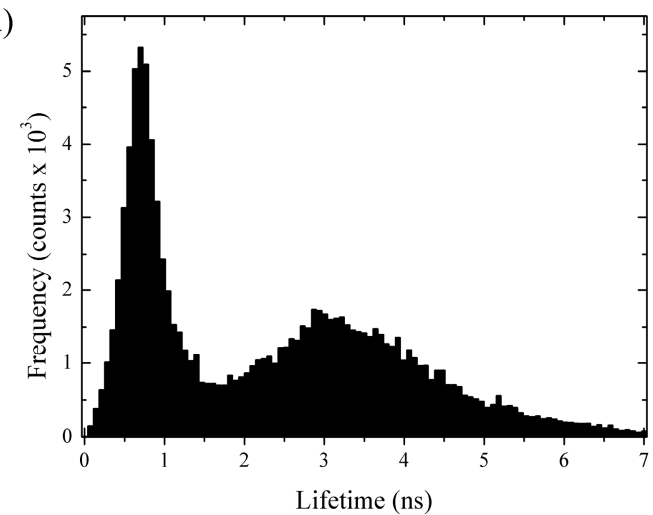

Fig. 4. FLIM image of an array of aluminosilicate microtubes in a microporous glass matrix (a) The image was collected at $250 \mathrm{x} 250$ pixel resolution with 4096 time channels; $2 \mathrm{~ms}$ acquisition time per pixel and total recording time 6.63 min. Image size: $29.90 \mu \mathrm{m}$ $29.90 \mu \mathrm{m}$. Panel (b) shows an enlarged image of one single aluminosilicate microtube in a glass matrix (indicated by frame in panel (a)). In this case the image was collected at the same pixel resolution and recording time, with image size $7.30 \mu \mathrm{m} \times 7.30 \mu \mathrm{m}$. Panels (c) and (d) demonstrate the corresponding lifetime histograms.

In contrast to the broad PL band in the spectra of tubes embedded in a microporous glass matrix (Figure 3a), the emission spectra of a single "free standing" microtube exhibit very sharp periodic structure (Figure 6). When separated from the matrix, the microtube is much more optically dense than its surrounding medium allowing light propagating inside the microtube to be spatially constrained to travel along the rim of a cross-section of the microtube, and therefore it is said to be trapped in a WGM. The presence of sharp emission peaks in the spectrum of a single microtube is an immediate result of this optical confinement (Figure 6). These peaks correspond to optical resonance locations and reflect the fact that transition probabilities are increased for emission wavelengths near resonance.

The WGM peaks are superimposed on a background signal arising from a part of the emission, which does not match any WGM of the microtube (Figure 6). 

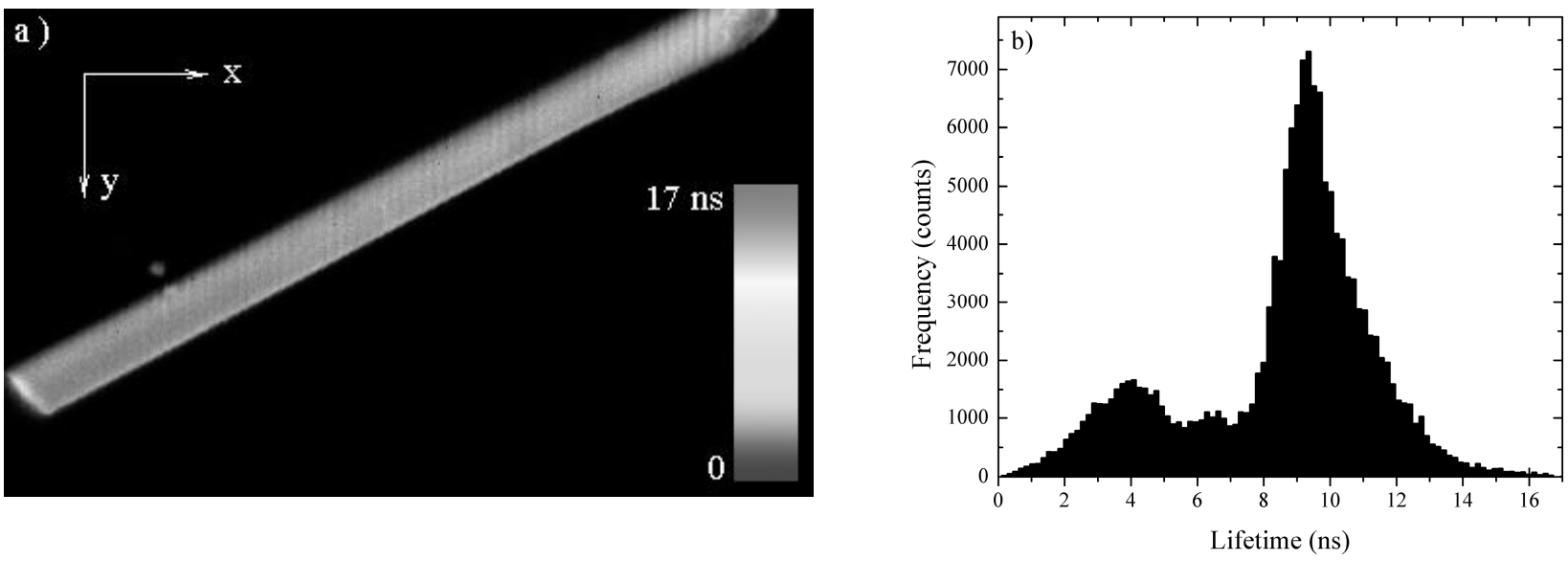

Fig. 5. FLIM image of a single aluminosilicate microtube (a). The image was collected at 416x250 pixel resolution with 4096 time channels; 2 ms acquisition time was provided per pixel and total recording time was 9.6 min. Image size: $67.72 \mu \mathrm{m}$ x $40.70 \mu \mathrm{m}$. Right panel shows the lifetime histogram. Every pixel in the lifetime image (a) gives the lifetime at that particular position in space (x,y).

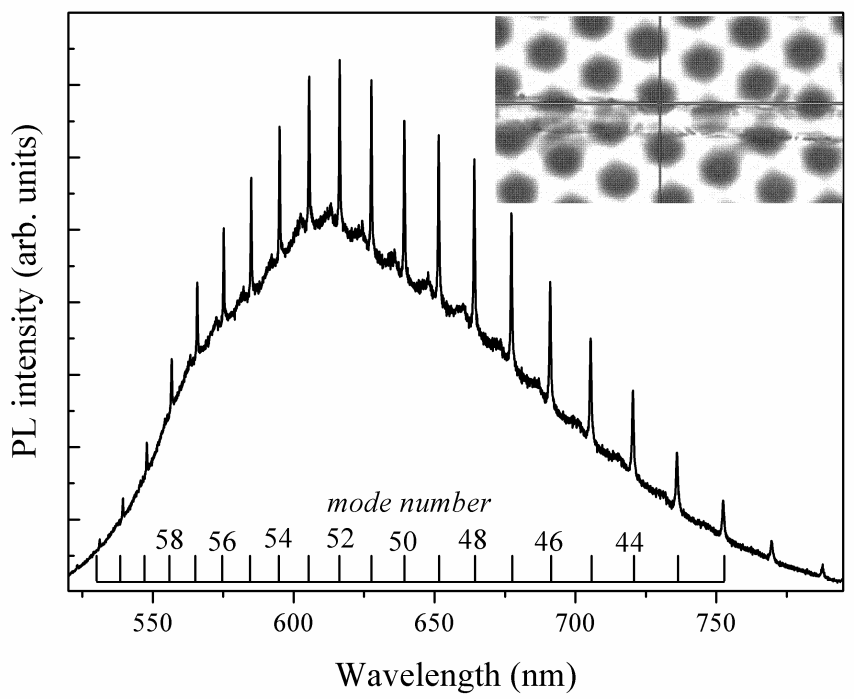

Fig. 6. Room-temperature PL spectra of a single free-standing microtube. The inset shows a microscope image of the microtube, with the cross-hairs indicating the excitation-detection position.

In the small size regime for microcavities $(<10 \mu \mathrm{m})$ where polarized modes are well separated, the number of modes is small and they can be identified. For studies of lasing and quantum information processing in particular, there is a need to determine the mode number and polarization of the WGM resonances. In order to identify the peaks in the observed WGM structure, we have adopted the boundaryvalue solution to the problem of scattering of plane electromagnetic waves by a dielectric micro-cylinder. ${ }^{30,31}$ The mode identification takes account of the cylindrical symmetry of the microtubes. The extinction efficiency $\mathrm{Q}$ is derivable from Lorenz-Mie theory when the microcylinder is illuminated by monochromatic, plane polarized light with the electric vector vibrating parallel to the cylinder axis (TM polarization):

$$
Q_{e x t}^{T M}=\frac{2}{x} \operatorname{Re}\left[b_{0}+2 \sum_{n=1}^{\infty} b_{n}\right],
$$

or for the case when the electric field is linearly polarized normal to the axis of the cylinder (TE polarization): 


$$
Q_{e x t}^{T E}=\frac{2}{x} \operatorname{Re}\left[a_{0}+2 \sum_{n=1}^{\infty} a_{n}\right],
$$

where $x=2 r / \lambda$ is the size parameter, $r$ is the radius of microcylinder, $n$ is the angular mode number. Apart from $n$ (which is proportional to the circumference of crosssection divided by the wavelength of the light propagating within the microcylinder), the spectral distributions of TM and TE WGM are characterized by the mode order $\ell$ (which indicates the number of maxima in the radial distribution of the internal electric field), and the azimuthal mode number $m$ (which gives the orientation of the WGMs orbital plane). ${ }^{32}$

In the absence of gain, the Mie scattering partial wave amplitudes an $(\mathrm{x}, \mathrm{m})$ and $\mathrm{bn}(\mathrm{x}, \mathrm{m})$ can be expressed in the form

$$
a_{n}=\frac{J_{n}^{\prime}(m x) J_{n}(x)-m J_{n}(m x) J_{n}^{\prime}(x)}{J_{n}^{\prime}(m x) H_{n}^{(1)}(x)-m J_{n}(m x) H_{n}^{\prime(1)}(x)}
$$

for TE modes and

$$
b_{n}=\frac{m J_{n}^{\prime}(m x) J_{n}(x)-J_{n}(m x) J_{n}^{\prime}(x)}{m J_{n}^{\prime}(m x) H_{n}^{(1)}(x)-J_{n}(m x) H_{n}^{\prime(1)}(x)}
$$

for TM modes, where $J_{n}(x)$ is a Bessell function of the first kind, $H_{n}(x)$ is a Henkel function of the second kind, and the primes denote differentiation of the functions with respect to their arguments. According to Eqs. (1)-(2) resonance structure in scattering spectra can be expected as the real part of an or bn reaches its maximum value of 1 and the imaginary part is passing through 0 from the positive to the negative side. In other words, the resonances in the Mie scattering characteristics occur when denominators in Eq. (3) (TE modes) or Eq (4) (TM modes) are equal to zero for a particular mode $n$.

These conditions are transcendental equations, which can be solved for the size parameter $x$ (position of resonance) for given values of refractive index and for given mode number $n$. Thus, comparing the calculated results with the spectral positions and polarization of the WGM in the experimental PL spectra, we can identify the indexes $n$ for each mode using the diameter of the microtube that was found from the SEM micrographs.

The algorithm of the mode assignment can be as follows: 1) The resonant wavelengths corresponding to WGM resonances $\lambda_{i}^{\text {exp }}(i=1,2 \ldots, N)$ are determined from a PL spectrum of single microtube; 2$)$ We assume an approximate value of the microtube radius based on the cross-sectional SEM image; 3) Theoretical resonance positions $\lambda_{i}^{\text {theor }}$ are then calculated using eqs. (3) and (4); 4) The two lists are compared and for each value of $\lambda_{i}^{\text {exp }}$, the closest value $\lambda_{i}^{\text {theor }}$ is determined and the difference between them $\Delta_{\mathrm{i}}$ is calculated and 5) Taking into account the spectral resolution $\Delta$, the correlation $\mathrm{S}=\mathrm{N}^{-1} \Sigma\left(1+\Delta_{\mathrm{i}} / \Delta\right)^{-1}$ is then maximized by adjusting only two fitting parameters, namely the size of the microcylinder and the refractive index. The results of the mode identification $\left(T M_{n}^{l}\right)$ for refractive index $=1.48$ and $\mathrm{D}$ $=7.65 \mu \mathrm{m}$ are shown in Figure 6. Agreement with the experimental values is excellent.

The procedure for WGM identification allowed us to reveal another striking feature of the spectra of a single microtube strong polarization properties. Experimentally, the distinction between modes of TE and TM polarization can be determined utilizing a polarizer inserted into the optical beam path in front of the detection system, which selects only the component of the electro-magnetic field parallel to the orientation of the polarizer. The sharp dominating peaks in the spectrum for polarizer orientation parallel to the microtube axis (Figure 7, curve 1) correspond to linear polarized light with the electric vector vibrating parallel to the axis of microtube. Rotating the polarizer by $90^{\circ}$ results in strong quenching of these WGMs (Figure 7, curve 2) unambiguously indicating their TM character.

A close look at the spectra presented in Figures 6 and 7 reveals the presence of satellites, broader peaks of the same polarization, which are blue-shifted with respect to the identified WGMs (indicated by arrows in Figure 7). The separation between these satellites homogeneously increases with wavelength ranging between 8 and $18 \mathrm{~nm}$ and is identical to the WGM spacings. Therefore, the observed secondary structure cannot be attributed to the WGM of higher $l$, for which smaller mode spacings are expected. Observation of two resonances of the same mode type can be explained by taking into account the fact that modes other than WGM can be supported by the microtube cavity. The presence of the second, inner surface in the microtube has a significant influence on the emission pattern of such microcavity systems. As a result, the microtube mode structure is more complex than in a single boundary microcavity such as a disc. Along with WGM originating from total internal reflection at the outer boundary there will be a different kind of mode 
penetrating the inner region at specific values of inner and outer diameter and refractive index. ${ }^{4}$ Weaker photon confinement will led to enhanced losses and therefore to a broadening of these modes.

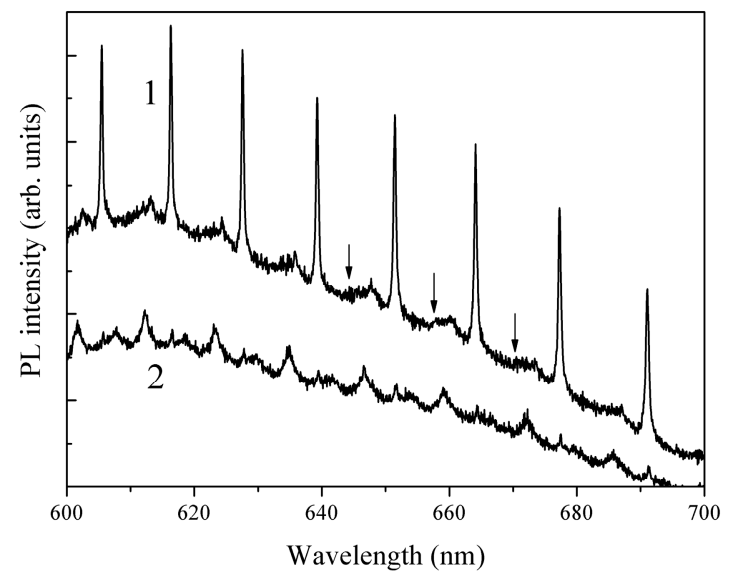

Fig. 7. PL spectra of single free-standing microtube recorded (1) with polarizer orientation parallel to the microtube axis and (2); with polarizer rotated by $90^{\circ}$.

If we fit the WGM peaks by a Lorentzian function, we find quality factors defined by $\mathrm{Q}=\lambda_{0} / \Delta \lambda$ ranging between 2000 and 3200 with the maximum Q-value obtained for the peak centered at $616 \mathrm{~nm}$. The quality factor reflects how long a photon can be stored in the microcavity before leaking out. Since the cavity $Q$ factor is directly related to the lifetime of the photon in the cavity $\tau_{\text {cav }}=\mathrm{Q} / \omega_{0}$ (where $\omega_{0}$ is the resonant frequency), we can estimate a time constant $\tau_{\text {cav }} \approx 1 \mathrm{ps}$. It is noteworthy that $\mathrm{Q}$ values obtained at room temperature for the investigated microtube resonators are about the same magnitude as those recently reported for low-temperature $(\mathrm{T}=5 \mathrm{~K})$ spectra of semiconductor microtube ring resonators fabricated by optical lithography and wet-etching processes. ${ }^{22}$ This is the highest Q-factor achieved to date in the spectra of micro-cylinders or micro-tubes with diameter comparable to the present samples.

To monitor the surface quality and homogeneity of the fabricated microtube we have studied the micro-PL spectra obtained for different positions of the exciting laser spot along the microtube axis. It turned out that the WGM positions shift less than $2 \mathrm{~nm}$ over a distance of $10 \mu \mathrm{m}$. From our calculations, we have determined that this shift is a result of a difference in microtube diameter of only $20 \mathrm{~nm}$.

It is well known that the resonant internal field of a microcavity is not completely confined to the interior of the microresonator. Depending on the shape and size of the microcavity, the evanescent field can extend into the surroundings up to a couple of microns. It was recently recognized that the partial delocalization of Mie resonance states is of great importance, because it suggests the possibility for coherent coupling between WGMs of two adjacent microcavities with closely matched sizes. ${ }^{33,34}$ For both TE and TM polarizations, it was theoretically shown that the contribution of a resonant mode to the field outside the microcylinder decays exponentially with steeper decay predicted for the lower value of mode order $l .{ }^{35}$ In this present work we experimentally examine the distribution of this evanescent field.

In Figure 8a, the integrated PL efficiency of a single microtube cavity is shown versus the excitation beam position scanning in the direction perpendicular to the microtube axis.

It is clearly seen than the localization of the focal spot at the rim of the microtube (as compared to the central excitation) leads to an increase in integrated PL intensity mostly due to the glancing angle-of-incidence excitation with respect to the cavity surface. This effect is accompanied by 3 -times increase in WGM visibility, which can be defined as $\mathrm{V}=\left(\mathrm{I}_{\text {peak }}-\right.$ $\left.\mathrm{I}_{\text {background }}\right) /\left(\mathrm{I}_{\text {peak }}+\mathrm{I}_{\text {background }}\right.$ ) (where $\mathrm{I}_{\text {peak }}$ and $\mathrm{I}_{\text {background }}$ are the intensities of WGM and background PL, respectively) (Figure 8b).

It should be particularly emphasized that the WGM structure observed in the PL spectra of a single aluminosilicate microtube is governed by the optical properties of the microcavity and would not be radically altered in scattering spectra, lasing, Raman or stimulated Raman scattering in the same way that in a semiconductor laser the lasing does not alter the cavity resonances. The results presented in Figure 8 clearly demonstrate that strength and efficiency of the WGM are highly dependent on the excitation location. The evanescent field associated with cavity modes extends into 
the surroundings up to a distance comparable to the external radius of the microtube. This observation, along with selective control of WGM visibility, suggests a possibility to achieve strong evanescent coupling between adjacent high$Q$ microresonators, and therefore, implies manifold applications, in biosensing, optical buffering and quantum information processing.

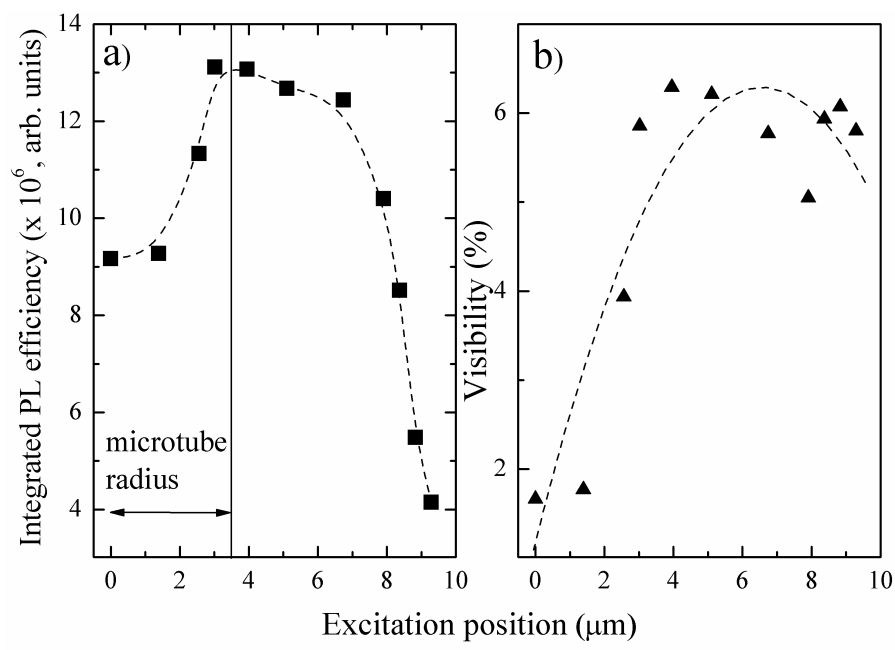

Fig. 8. (a) Total luminescence intensity versus excitation beam position scanning in direction perpendicular to the microtube axis and (b) visibility of $T M_{52}$ WGM. The zero position corresponds to the excitation point located on the axis of the microtube.

As a result of the high quality factor and the very narrow WGM peaks along with the cylindrical geometry that is useful for optical pumping, microtube cavities are very promising systems for the design of an optically pumped microlaser emitting at room temperature.[3] In order to understand the emission process further, we have studied the micro-PL spectra and lifetime of the PL as a function of intensity $\left(I_{\text {pump }}\right)$ (Figure 9$)$.

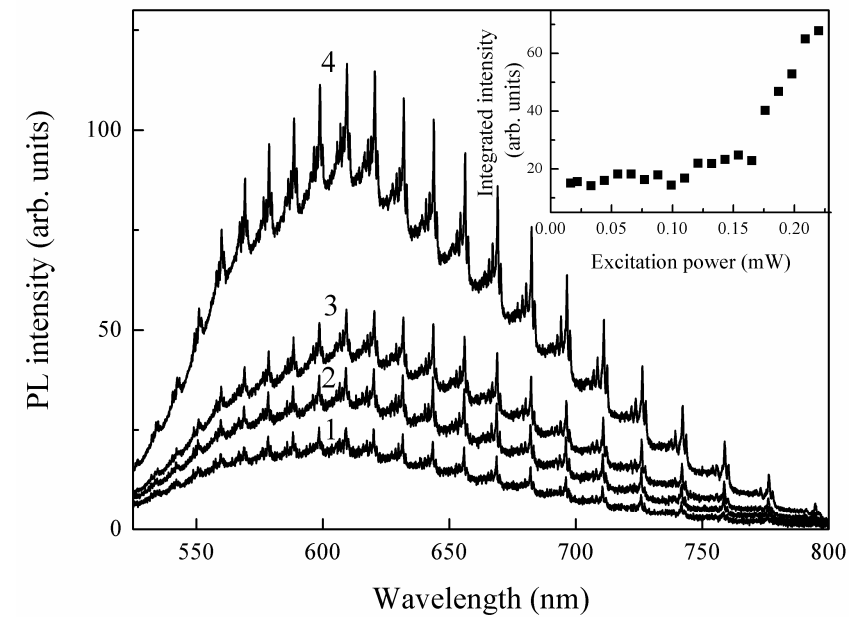

Fig. 9. Power-dependent PL spectra of a single aluminosilicate microtube excited by Ar+ laser $\left(\lambda_{\text {ex }}=514.5 \mathrm{~nm}\right)$ at power $0.02 \mathrm{~mW}$ (Curve 1); $0.06 \mathrm{~mW}$ (Curve 2); $0.09 \mathrm{~mW}$ (Curve 3) and $0.22 \mathrm{~mW}$ (Curve 4). The inset shows the dependence of the integrated PL emission on excitation power, which was measured at the excitation spot.

Time-resolved studies in the literature have focused on the modification of the spontaneous recombination dynamics in 3D microcavities, ${ }^{37-41}$ or investigation of cavity enhanced excitation energy transfer. ${ }^{37,42}$ Knowledge of the emission dynamics under amplification conditions should provide important information for the design of 3D microcavity laser devices. ${ }^{2,43}$ Amplified spontaneous emission shows itself as an acceleration of the PL decay and therefore a powerdependent shortening in the emission lifetime unambiguously indicates the transition from spontaneous emission to lasing. 
The results presented in Figure 10 confirm that an increase of excitation power leads to an acceleration of the emission decay in the single microtube cavity. The recorded distributions of lifetimes are very reproducible going back to the low excitation level and the original PL decay characteristics are restored. Moreover, from the data presented in Figure 10 we can conclude that although the PL efficiency at high powers is affected to some extent by photodegradation, it does not lead to a significant modification of the recombination dynamics. The clear decrease in the PL lifetime (Figure 10) lends strong credence to the occurrence of amplified spontaneous emission in the single microtube. The acceleration of the emission decay observed here is due to increased gain in the aluminosilicate glass as an active material and can be efficiently controlled by excitation power. It is noteworthy that the shortening of the decay times for higher excitation power cannot be caused by a heating effect. Any heating of microtubes will be accompanied by a spectral shift of the optical modes in the spectra, however in our experiments the spectral positions of the WGM peaks were unchanged with increasing excitation power.

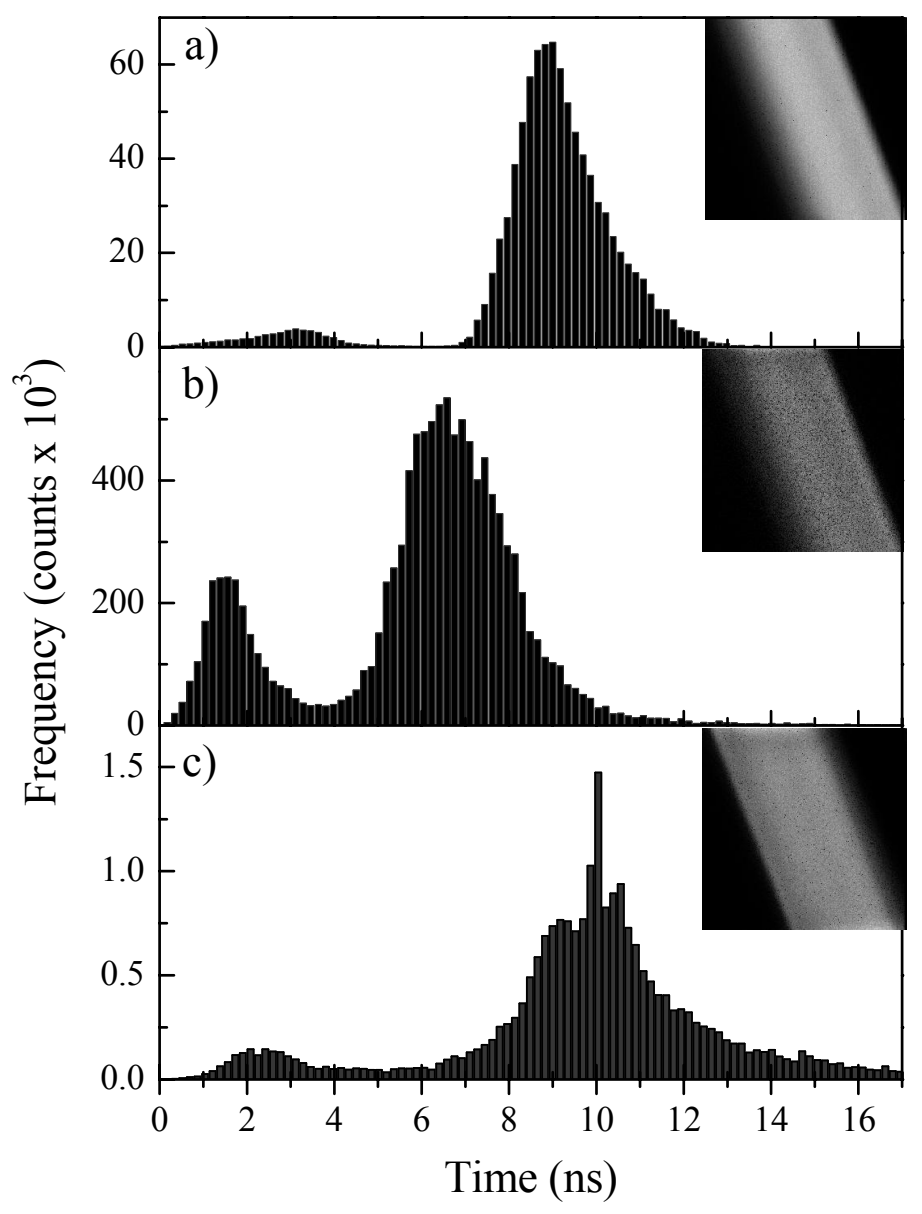

Fig. 10. PL lifetime histograms and the corresponding FLIM images (insets) of a single aluminosilicate microtube at varying excitation powers. Insets in the right panel show the corresponding FLIM images of $9.60 \times 9.60 \mu \mathrm{m}$ size collected at $250 \times 250$ pixel resolution, $2 \mathrm{~ms} /$ pixel acquisition and total recording time $6.55 \mathrm{~min}$.

\section{CONCLUSIONS}

Thus we have developed a simple and robust technique for fabricating luminescent aluminosilicate microtubes and we have demonstrated the high-Q resonance modes in the emission spectra of a single microcavity. The method of preparation of such structures has unique advantages providing high-quality small three-dimensional microcavities with strongly polarized emission. The large evanescent field associated with cavity modes was found to extend into the surroundings up to a distance comparable to the external radius of the microtube. The pump-power dependence of the microtube emission and the strong acceleration of the emission decay at high excitation power confirm the occurrence of 
amplified spontaneous emission from a single microtube. Potential applications for these alumosilicate microresonators will be in the areas of microlasers and microsensors and quantum information processing.

\section{REFERENCES}

1. W. von Klitzing, R. Long, V. S. Ilchenko, J. Hare, V. Lefèvre-Seguin, "Tunable whispering gallery modes for spectroscopy and CQED experiments", New. J. Phys. 3, 14.1-14.4 (2001).

2. K. J. Vahala, "Optical microcavities", Nature 424, 839-846 (2003).

3. H.-J. Moon, Y.-T. Chough, K. An, "Cylindrical Microcavity Laser Based on the Evanescent-Wave-Coupled Gain", Phys. Rev. Lett. 85(15), 3161- 3164 (2000).

4. H.-J. Moon, G.-W. Park, S.-B. Lee, K. An, J. H. Lee, "Laser oscillations of resonance modes in a thin gain-doped ring-type cylindrical microcavity", Opt. Commun. 235(4-6), 401-407 (2004).

5. S. Arnold, "Microspheres, Photonic Atoms and the Physics of Nothing", Am. Sci. 89(5), 414-421 (2001).

6. A. B. Matsko, V. S. Ilchenko, "Optical resonators with whispering-gallery modes-part I: basics", IEEE J. Sel. Top. Quantum Electron. 12(1), 3-14 (2006).

7. V. S. Ilchenko, A. B. Matsko," Optical resonators with whispering-gallery modes-part II: applications", IEEE J. Sel. Top. Quantum Electron. 12(1), 15-32 (2006).

8. Y. P. Rakovich, J. F. Donegan, N. Gaponik, A. L. Rogach, "Raman scattering and Anti-Stokes emission from a single spherical microcavity with a CdTe quantum dot monolayers", Appl. Phys. Lett. 83(13), 2539-2541 (2003).

9. R. A. Wallingford, A. G. Ewing, "Capillary zone electrophoresis with electrochemical detection in $12.7 \mu \mathrm{m}$ diameter columns", Anal. Chem. 60, 1972-1975 (1988).

10. S. Blair, Y. Chen, "Resonant-enhanced evanescent-wave fluorescence biosensing with cylindrical optical cavities", Appl. Opt. 40(4), 570-582 (2001).

11. K. Mitsuhashi, N. Tagami, K. Tanabe, T. Ohkubo, H. Sakai, M. Koishi, M. Abe," Synthesis of Microtubes with a Surface of "House of Cards" Structure via Needlelike Particles and Control of Their Pore Size", Langmuir 21(8), 3659$3663(2005)$.

12. R. M. Penner, C. R. Martin, "Preparation and electrochemical characterization of ultramicroelectrode ensembles", Anal. Chem. 59(21), 2625- 2630 (1987).

13. J. Choi, G. Sauer, K. Nielsch, R. B. Wehrspohn, U. Gosele, "Hexagonally Arranged Monodisperse Silver Nanowires with Adjustable Diameter and High Aspect Ratio", Chem. Mater. 15(3), 776-779 (2003).

14. C. D. Merritt, B. L. Justus, "Fabrication of Microelectrode Arrays Having High-Aspect-Ratio Microwires", Chem. Mater. 15(13), 2520-2526 (2003).

15. S. A. Sapp, D. T. Mitchell, C. R. Martin," Using Template-Synthesized Micro- and Nanowires as Building Blocks for Self-Assembly of Supramolecular Architectures", Chem. Mater. 11(5), 1183- 1185 (1999).

16. Y. L. Tai, H. Teng," Template Synthesis and Electrochemical Characterization of Nickel-Based Tubule Electrode Arrays", Chem. Mater. 16(2), 338-342 (2004).

17. M. Wirtz, C. R. Martin," Template-Fabricated Gold Nanowires and Nanotubes", Adv. Mater. 15(5), $455-458$ (2003).

18. D. J. Monk, D. R. Walt," Fabrication of Gold Microtubes and Microwires in High Aspect Ratio Capillary Arrays", J. Am. Chem. Soc. 126(37), 11416- 11417 (2004).

19. F. Namavar, F. Lu, C. H. Perry, A. Cremins, N. M. Kalkhoran, R. A. Soref," Strong room-temperature infrared emission from Er-implanted porous Si", J. Appl. Phys. 77(9), 4813 (1995).

20. T. Kimura, A. Yokoi, H. Horiguchi, R. Saito, T. Ikoma, A. Sato," Electrochemical Er doping of porous silicon and its room-temperature luminescence at $\sim 1.54 \mu \mathrm{m} "$, Appl. Phys. Lett. 65(8), 983-985 (1994).

21. A. M. Dorofeev, N. V. Gaponenko, V. P. Bondarenko, E. E. Bachilo, N. M. Kazuchits, A. A. Leshok, G. N. Troyanova, N. N. Vorosov, V. E. Borisenko, H. Gnaser, W. Bock, P. Becker, H. Oechsner, "Erbium luminescence in porous silicon doped from spin-on films", J. Appl. Phys. 77(6), 2679- 2683 (1995).

22. T. Kipp, H. Welsch, C. Strelow, C. Heyn, D. Heitmann, "Optical Modes in Semiconductor Microtube Ring Resonators", Phys. Rev. Lett. 96(7), 077403 (2006).

23. M. Steinhart, J. H. Wendorff, A. Greiner, R. B. Wehrspohn, K. Nielsch, J. Schilling, J. Choi, U. Gosele," Polymer Nanotubes by Wetting of Ordered Porous Templates", Science, 296(5575), 1997 (2002).

24. W. H. Green, K. P. Le, J. Grey, T. T. Au, M. J. Sailor," White Phosphors from a Silicate-Carboxylate Sol-Gel Precursor That Lack Metal Activator Ions", Science 276, 1826-1828 (1997).

25. M. R. Eftink, in Topics in Fluorescence Spectroscopy, Vol. 2, Plenum Press, New York, 1991. 
26. J.N. Demas, B.A. DeGraff, "Luminescence-based sensors: microheterogeneous and temperature effects", Sens. Act. $B$ 11, 35 (1993).

27. D.R. James, Y.-S. Liu, P. De Mayo, W.R. Ware," Distributions of fluorescence lifetimes: consequences for the photophysics of molecules adsorbed on surfaces", Chem. Phys. Lett. 124, 460 (1985).

28. E.R. Carraway, J.N. Demas, B.A. DeGraff," Luminescence Quenching Mechanism for Microheterogeneous Systems", Anal. Chem. 63(4), 332-336 (1991).

29. T. Förster," Transfer mechanisms of electronic excitation", Disc. Faraday Soc. 27, 7-17 (1959).

30. H.C. Van de Hulst, Light Scattering by Small Particles, John Wiley \& Sons, New York, 1957.

31. M. Kerker, E. Matijevic," Scattering of Electromagnet Waves from Concentric Infinite Cylinders", J. Opt. Soc. Am. 51, 506-508 (1961).

32. P.W. Barber, S.C. Hill, Light scattering by particles: computational methods, World Scientific, Singapore, 1990.

33. S. Deng, W. Cai, V. Astratov, "Numerical study of light propagation via whispering gallery modes in microcylinder coupled resonator optical waveguides", Opt. Express, 12(26), 6468-6480 (2004).

34. Y.P. Rakovich, J.F. Donegan, M. Gerlach, A.L. Bradley, T.M. Connolly, J.J. Boland, N. Gaponik, A. Rogach, "Fine structure of coupled optical modes in photonic molecules", Phys. Rev. A 70(5), 051801 (2004).

35. J.F. Owen, R.K. Chang, P.W. Barber, "Internal electric field distributions of a dielectric cylinder at resonance wavelengths", Opt. Lett. 6(11), 540-542 (1981).

36. K. Iga, Handbook of Seemiconductor Lasers and Photonic Integrated Circuits, Chapman\&Hall, London, 1994.

37. Y.P. Rakovich, M. Gerlach, A.L. Bradley, J.F. Donegan, T.M. Connolly, J.J. Boland, M. A. Przyjalgowski, A. Ryder, N. Gaponik, A.L. Rogach, "Confined optical modes in small photonic molecules with semiconductor nanocrystals", J. Appl. Phys. 96(11), 6761-6765 (2004).

38. G.C. Righini, C. Arnaud, S. Berneschi, M. Bettinelli, M. Brenci, A. Chiasera, P. Feron, M. Ferrari, M. Montagna, G. Nunzi Conti, S. Pelli, H. Portales, C. Siligardi, A. Speghini, L. Zampedri, "Integrated optical amplifiers and microspherical lasers based on erbium-doped oxide glasses", Opt. Mater. 27(11), 1711-1717 (2005).

39. U. Woggon, R. Wannemacher, M. Artemyev, B. Möller, N. Lethomas, V. Anikeyev, O. Schops, "Dot-in-a-dot: electronic and photonic confinement in all three dimensions", Appl. Phys. B 77(5), 469-484 (2003).

40. X.D. Fan, M.C. Lonergan, Y.Z. Zhang, H.L. Wang, "Enhanced spontaneous emission from semiconductor nanocrystals embedded in whispering gallery optical microcavities", Phys. Rev. B 64(11), 115310 (2001).

41. V. Zwiller, S. Fälth, J. Persson, W. Seifert, L. Samuelson, G. Björk, "Fabrication and time-resolved studies of visible microdisk lasers", J. Appl. Phys. 93(4), 2307-2309 (2003).

42. T. Takahashi, K. Fujiwara, S. Matsuo, H. Misawa, "Excitation energy transfer between dye molecules in lasing microparticles", J. Photochem. Photobiol. A 120, 135-140 (1999).

43. D. Klotzkin, P. Bhattacharya, "Bringing quantum dots up to speed [Breaking the phonon bottleneck with high-speed modulation of quantum-dot lasers]", IEEE Circuits \& Devices, 16, 17-23 (2000).

44. M. Nogami, Y. Abe, "Properties of sol-gel-derived $\mathrm{Al}_{2} \mathrm{O}_{3} / \mathrm{SiO}_{2}$ glasses using $\mathrm{Eu}^{3+}$ ion fluorescence", J. Non-Cryst. Solids, 197(1), 73-78 (1996). 